\title{
Photonic band gap structure for improve the Performance of Band stop filters
}

\author{
Pooja Sahoo ${ }^{1}$ and P.K. Singhal ${ }^{2}$ \\ ${ }^{1,2}$ Department of Electronics \& communication Engineering, Madhav Institute of Technology \& Science, \\ Gwalior (M.P), India \\ E-mail: ${ }^{1}$ sahu.pooja2009@gmail.com
}

\begin{abstract}
In this paper we have designed two new compact Photonic Band Gap Structures, shows the properties of Band stop filters. Photonic Band gap filters are designed on central frequencies $1.7 \mathrm{GHz}$ and $2.2 \mathrm{GHz}$. These Photonic filters are stable and observable. Also used photonic band gap structure on the filter surfaces, have been provided information about the increasing bandwidth up to $30 \%$ of band stop filters. To use of the PBG filters, the broad stop band has been obtained, slow wave factor increased, com-pensate for fabrication of tolerance has been improved and the unit elements of the band stop filter with open-circuited stub has been redundant and more compact configuration obtained.
\end{abstract}

Keywords: PBG (Photonic Band Gap), Band Stop Filter (BSF), Printed Circuit Board (PCB), Defected Ground Structure (DGS), Computer Simulation Technology Software (CST) and Spectrum Analyzer.

\section{INTRODUCTION}

The photonic band-gap (PBG) [Yu chen et al., 2008] structures are effective in microwave applications that provide an effective control of electromagnetic (EM) waves along specific direction and performance. Photonic band gap structures for microstrip line have been topic of research in recent year. The term PBG is introduced as a structure which influence or even changes the electromagnetic properties of materials. The periodic structure created in materials such as substrate or metals [yang et al. 1999]. The PBG structure provides a certain frequency bands which cannot propagate. PBG structures are most widely used in various applications like microwave filters, antenna and other devices. The different structures LPF, BSF, power divider, power amplifier etc. may be implemented [Oraizi et al.2008]. In addition to DGS (defected ground plane) and EBG (electromagnetic band gap) structure, PBG have been created by etching different shapes in ground plane, which increase the inductance and capacitance values of microstrip line. The above technique used for eliminate undesired output response and sharp stop band for LPF [Shao, 2005; Atallah ali, 2007].

Dr. D’Orazio [1998] has been fabricated the PBG filter for wavelength division multiplexing (WDM) [1] \& Villar [1999] has been analyzed the PBG structure with a liquid crystal defect for the purpose of fiber optic filters [M. S. Esfahlan, 2008].

The basic phenomena behind the proposed photonic band gap filter are forbidden gap in materials by electrons movement [Lancaste, book]. Recently a Photonic band gap structure consisting of small metal pads with grounding via which used to improve the performance of a patch antenna [Y. D. Chen, 2008; Yongxi Qian,1999]. The most important function of PBG structures is the filtering of frequency bands, and harmonics of the filter in microwave circuit.

\section{BAND STOP FILTER DESIGN}

Design-a narrow-band Band stop filter with Lresonators. Five order microstrip band stop filter in Chebyshev prototype having pass band ripple of 0.1 $\mathrm{db}$. The desired band-edge frequencies to equalripple points are $f_{1}=4.5 \mathrm{GHz}$ and $\mathrm{f}_{2}=5.5 \mathrm{GHz}$. 
Choosing $\mathrm{Z}_{0}=50$ ohm, $\mathrm{g}_{0}=1.1468, \mathrm{~g}_{2}=1.3712$ $\mathrm{g}_{3}=1.9750 \mathrm{~g}_{4}=1.3712 \mathrm{~g}_{5}=1.1468 \mathrm{~g}_{6}=1$. Length of Lresonators $l_{\mathrm{h}}=8.9 \mathrm{~mm}$ and $\mathrm{l}_{\mathrm{v}}=8.9 \mathrm{~mm}$ half guided wavelength Spacing of main line and resonators $\mathrm{s}_{1}=\mathrm{s}_{5}=0.292 \mathrm{~mm}, \quad \mathrm{~s}_{2}=0.292 \mathrm{~mm}, \quad \mathrm{~s}_{3}=0.292 \mathrm{~mm} \quad \&$ $\mathrm{s}_{4}=.292 \mathrm{~mm}$.

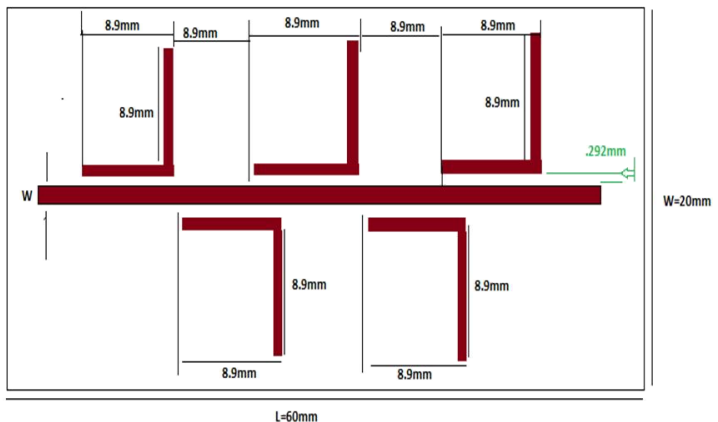

Fig. 1. (a) L-resonator narrowband Band stop filter

Design- an optimum microstrip band stop filter with three open-circuited stubs and FBW $=1.0$ at a mid-band frequency $\mathrm{f}_{0}=2.5 \mathrm{GHz}$. Now assuming a pass band return loss of $-20 \mathrm{~dB}$, which corresponds to a ripple constant $\varepsilon=0.1005$ and Choosing $Z_{0}=$ $50 \mathrm{ohm}$. The optimum band stop filter is synthesized using optimum transfer function Chebyshev functions of first kinds order $\mathrm{n}$

$\operatorname{Tn}(x)=\cos \left(n \cos ^{-1} x\right)$

Chebyshev functions of second kinds order $\mathrm{n}$ $U n(x)=\sin \left(n \cos ^{-1} x\right)$

And calculation of parameters made by given equations:

$Z_{a}=Z_{b}=Z_{0}$

$Z_{i}=Z_{0} / g_{i}$

$Z_{i, t+1}=Z_{0} / J_{i, t+1}$

$t=j \tan \left(\frac{\pi}{2} \frac{f}{f_{0}}\right)$

Table 1: Parameters Calculation for Filter Design

\begin{tabular}{|l|l|l|}
\hline Impedance (ohm) & Width $(\mathrm{mm})$ & Length $(\mathrm{mm})$ \\
\hline $\mathrm{Z}_{\mathrm{a}}=\mathrm{Z}_{\mathrm{b}}=50$ & 1.85 & 5.475 \\
\hline $\mathrm{Z}_{1}=\mathrm{Z}_{3}=52.74$ & 1.7 & 14.32 \\
\hline $\mathrm{Z}_{2}=29.88$ & 4.25 & 13.73 \\
\hline $\mathrm{Z}_{12}=\mathrm{Z}_{23}=93.97$ & .528 & 14.89 \\
\hline
\end{tabular}

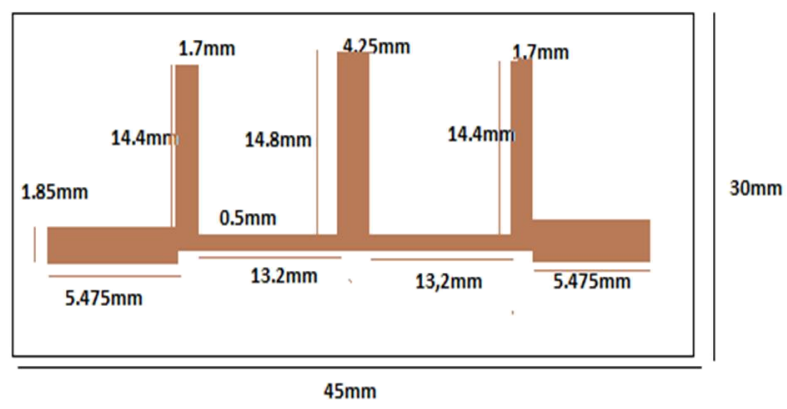

Fig. 2. (a) Layout of L-resonator band stop filter

The microstrip filters have been designed on a substrate which has thickness of $1.6 \mathrm{~mm}$ and a dielectric constant of 4.4. The open-end and Tjunction effects should also be taken into account for determining the final filter dimensions. The optimum design demonstrates substantially improved performance with a steeper stop band response.

Design-Fig 3(a) have been designed for improve the response of L-resonator band stop filter. It designed for a three order microstrip Band stop filter in Chebyshev prototype with Passband ripple of $0.05 \mathrm{db}$. The desired band-edge frequencies to equal ripple points are $\mathrm{f}_{1}=1.25 \mathrm{GHz}$ and $\mathrm{f}_{2}=3.75$ $\mathrm{GHz}$ and $\mathrm{Z}_{0}=50 \mathrm{ohm}$.

Equations for designing Band stop filter with open circuit stub are Calculation for cutoff frequency:

$f_{0}=\frac{f_{1}+f_{2}}{2}$

Calculation for Fractional Band Width

$F B W=\frac{f_{2}-f_{1}}{f_{0}}$

$g$-values of the prototype filter

$g_{0}=g_{4}=1.0, g_{1}=g_{3}=0.8794$

$g_{2}=1.1132, z_{a}=z_{b}=50$

Calculation for impendences

$Z_{1}=Z_{a}\left(1+\frac{1}{\propto g_{0} g_{1}}\right)$

$Z_{2}=\frac{Z_{a} g_{0}}{\propto g_{2}}$

$Z_{3}=\frac{Z_{a} g_{0}}{g_{4}}\left(1+\frac{1}{\propto g_{3} g_{4}}\right)$

$Z_{12}=Z_{a}\left(1+\propto g_{0} g_{1}\right)$

$Z_{23}=\frac{Z_{a} g_{0}}{g_{4}}\left(1+\propto g_{3} g_{4}\right)$

$\propto=\cot \left[\frac{\pi}{2}\left(1-\frac{F B W}{2}\right)\right]$ 
P. Sahoo and P.K. Singhal / International Journal of Computer Networks and Communications Security, 2 (2), February 2014

Table 2: Parameters Calculation for Filter Design

\begin{tabular}{|l|l|l|}
\hline Impedance(ohm) & Width(mm) & Length(mm) \\
\hline$Z_{\mathrm{a}}=\mathrm{Z}_{\mathrm{b}}=50$ & 1.85 & 5.475 \\
\hline $\mathrm{Z}_{1}=\mathrm{Z}_{3}=106.85$ & 0.3 & 15.15 \\
\hline $\mathrm{Z}_{2}=44.92$ & 2.3 & 14.85 \\
\hline $\mathrm{Z}_{12}=\mathrm{Z}_{23}=93.97$ & .45 & 14.05 \\
\hline
\end{tabular}

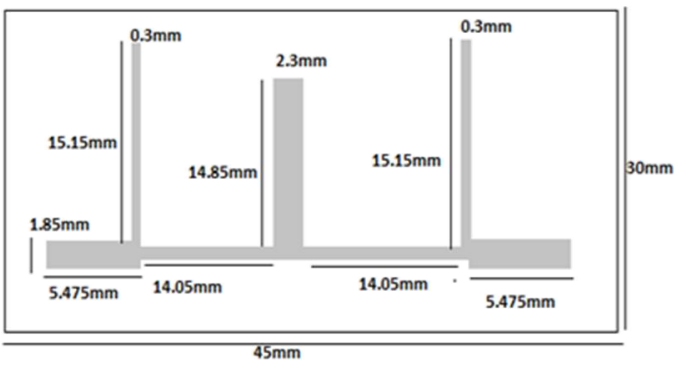

Fig. 3. (a) Open circuit Microstrip Band Stop Filter

\section{DESIGN OF PBG STRUCTURE}

PBG structures have been designed for unaltered the frequencies band for particular response of the filters. It has been designed back surface of the PCBs. Few photonic band gap (PBG) structures have been demonstrated on FR-4 substrate Pcbs with thickness of $1.6 \mathrm{~mm}$ and $\varepsilon_{\mathrm{r}}$ is 4.4 and $\mu-1$.

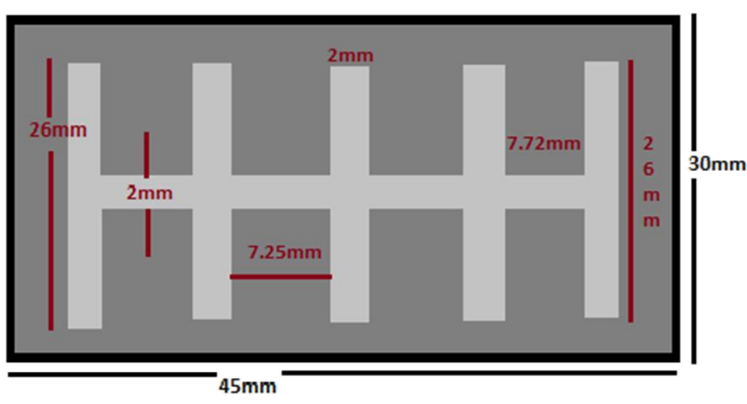

Fig. 4. (a) E-Shapes Photonic Band Gap Structure

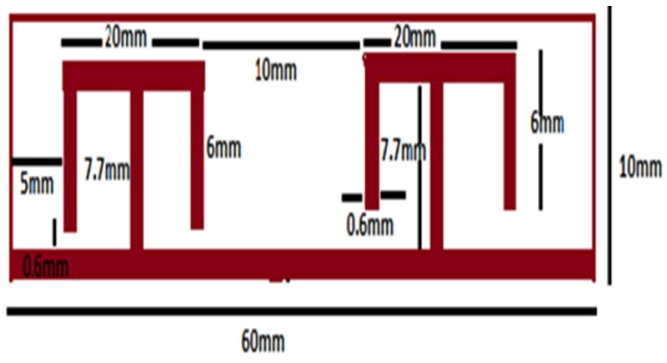

Fig. 5.(a) T Shape Photonic Band Gap Structure

\section{SIMULATED RESULT}

The simulated results of band stops filters with and without PBG structure are shown in Fig 1(b), 2(b), 3(b). Fig 1(b) at central frequency $5 \mathrm{GHz}$ provide very small band gap $0.7 \mathrm{GHz}$, which is approximate $14 \%$ of central frequency.

To improve the performance of L-resonator filter we used optimum band stop filter for wide stop band. Optimum filter response provide wide band gap $1.69 \mathrm{GHz}$ at central frequency $3 \mathrm{GHz}$. The band gap is $56.33 \%$ of central frequency as shown in Fig 2(b).

To reduced the side band fluctuation we designed open circuit band stop filter. Open circuit filters have very simple calculation for designing, the central frequency is $3.05 \mathrm{GHz}$ and band gap $1.815 \mathrm{GHz}$ which exactly $59.50 \%$ of central frequency shown Fig 3(b).

Now Fig 4(b) shows the response of photonic band gap filter provide two band gaps at central frequencies $2.2 \mathrm{GHz} \& 4.8 \mathrm{GHz}$. and Fig 5(b) shows another photonic band gap result at $1.7 \mathrm{GHz}$ and provide wide band gap $1.5 \mathrm{GHz}$. these photonic band gap filters are simpler than the open circuit band stop filter and easily designed.

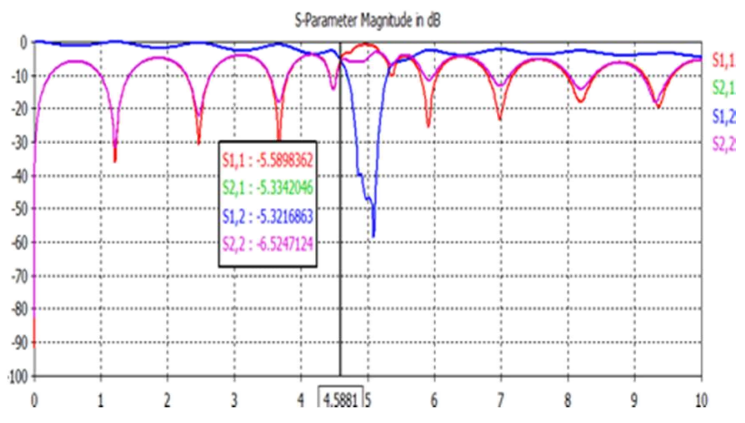

Fig. 1. (b) response of L-resonator band stop filter

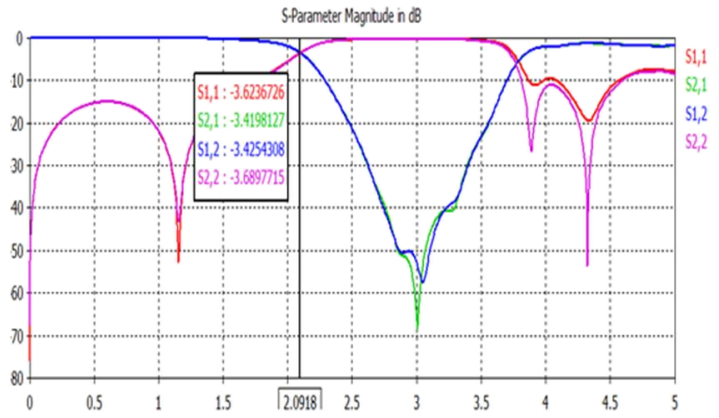

Fig. 2. (b) response of optimum band stop filter 
P. Sahoo and P.K. Singhal / International Journal of Computer Networks and Communications Security, 2 (2), February 2014

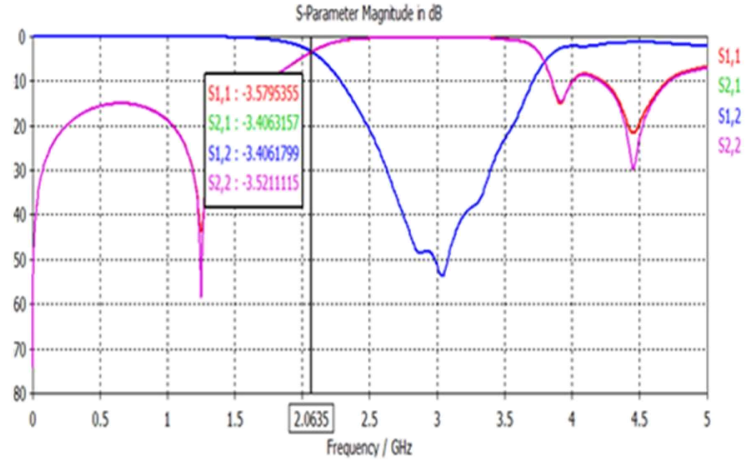

Fig. 3. (b) response of open circuited band stop filter

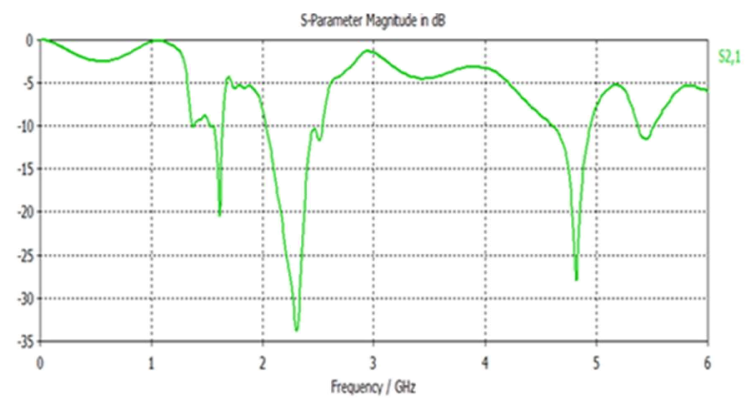

Fig. 4. (b) response of photonic band gap filter

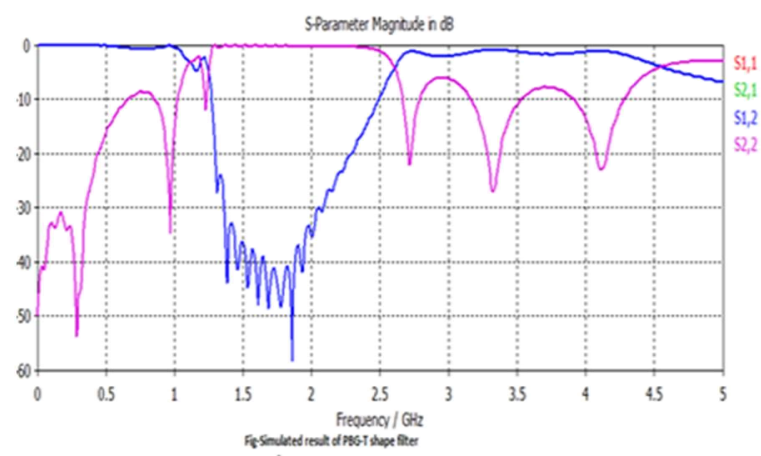

Fig. 5. (b) response of photonic band gap filter

Fig 6 shows the input output ports signals for each photonic band gap filters.

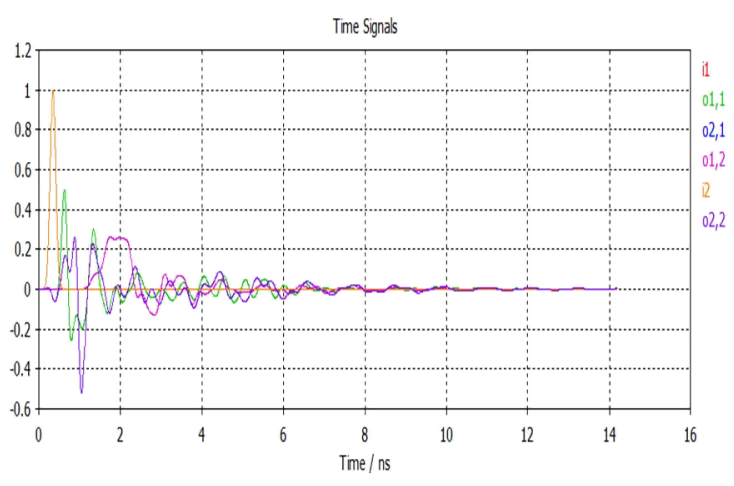

Fig. 6. I/O ports signals
Now smith charts for these PBG filter shown in fig 6(a) and Fig 6(b) for return loss nad insertion loss, provide the great stability for the filters. At $5 \mathrm{GHz}$ the total impedance is $(14.8,1.07) \mathrm{ohm}$ and $(19.8,68.6) \mathrm{ohm}$.

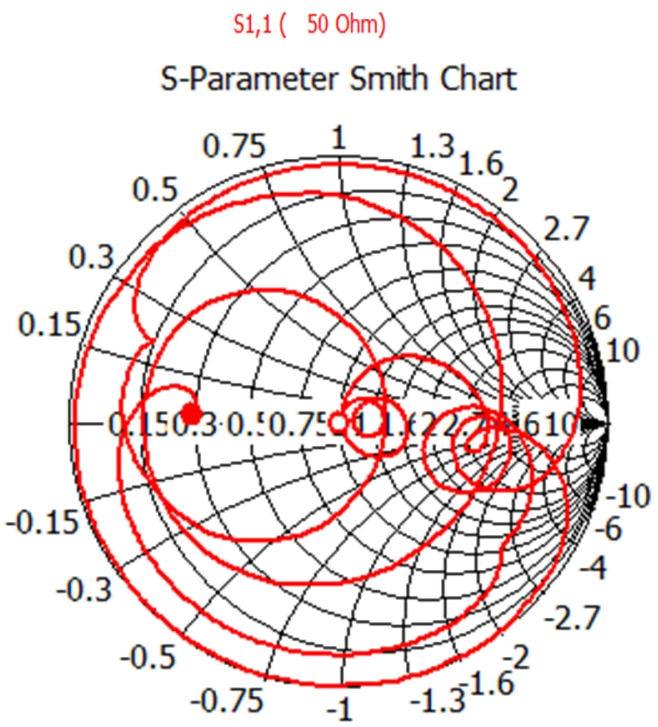

Fig. 6. (a) Smith chart for the proposed PBG Filter

$$
52,2(500 \mathrm{hm})
$$

S-Parameter Smith Chart

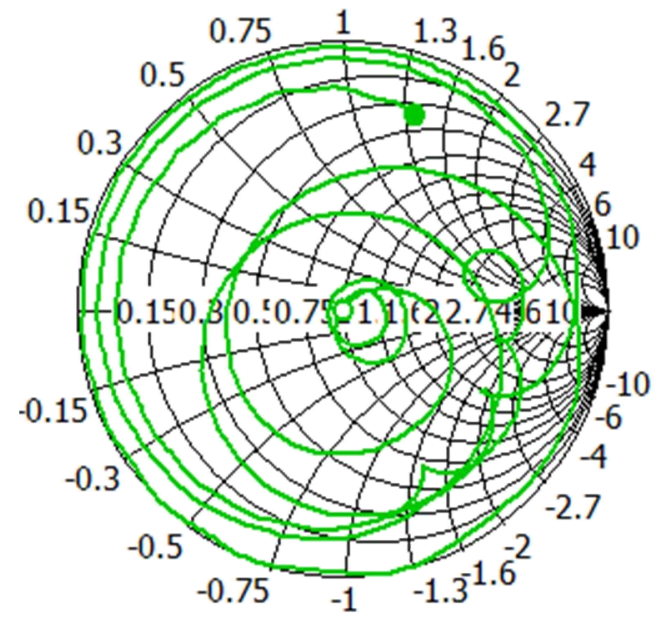

Fig. 6. (f) Smith chart for the proposed PBG Filter

\section{CONCLUSION}

Conclusion are made by the various responses obtained in fig 1(b) for L-resonator, fig 2(b) for optimum band stop filter\& fig 3(b) for open circuit band stop filter and various PBG structure response shown in fig4(b) and fig5(b) which have been designed for different cut off frequencies. These novel PBG structures have wide stop band and 
compact size, which can be easily incorporated into the ground planes of Pcbs or any other planer structures. The PBGs structures designing are very simpler than the design of band stop filters.

Results are simulated using computer simulation technology software (CST). The undesired sidebands and fluctuations of response are reduced by using PBG and improvement in bandwidth is achieved in case of band stop filter (BSF). This PBG structures should find wide applications for high-performance in microwave circuits.

\section{REFERENCES}

[1] Cheng-Hung Lin, Guan-Yu Chen, Jwo-Shiun Sun, Kwong-Kau Tiong1, and Y. D. Chen "The PBG Filter Design" PIERS Proceedings, Hangzhou, China, March 24-28, 2008

[2] J.-K.Xiao and Y.-F. Zhu "New U-Shaped DGS Bandstop Filters" progress in Electromagnetics Research C, vol.25, 179-191, 2012

[3] Fei-Ran Yang, Kuang-Ping Ma, Yongxi Qian "A Unipolar Compact Photonic Bandgap Structure And Its Applications For Microwave Circuits" IEEE Transactions on microwave theory and techniques, vol 47 , no. 8 , august 1999.

[4] H. Oraizi and m. S. Esfahlan "Miniaturization of Wilkinson Power Dividers by Using Defected Ground Structures" progress in Electromagnetics Research Letters, vol.4, 113120, 2008.

[5] Shao Ying Huang, Yee Hui Lee "Compact UShaped Dual Planar EBG Microstrip Low-Pass Filter" IEEE Transactions on Microwave Theory and Techniques VOL.53, No.12, Dec2005.

[6] Atallah Balalem, Ali, Jan Machac “ QuasiElliptic Microstrip Low-Pass Filters Using an Interdigital DGS"IEEE Microwave and Wireless Components letters 1531-1309 2007.

[7] Jia-Sheng Hong, M. J. Lancaste "Microstrip filters for RF / Microwave Application" A Wiley-Interscience Publivation book.

[8] Lakhan Singh and P. K. Singhal "Design And Comparision Of Band Pass Trisection Microstrip Filter" International Journal of Engineering Research \& Technology (IJERT),Vol. 2 Issue 2, February- 2013, ISSN: 2278-0181.

[9] CST (computer Simulation Technology) software microwave studio 2010. 